\title{
Editorial
}

\section{Chironomidae and the tree of life}

The importance of understanding the genealogical relationships between populations, species and species groups can hardly be exaggerated. As reconstructions of evolutionary pathways through time, phylogenetic trees provide a framework on which biological hypotheses can be tested; without robust phylogenies we will be unable to fully understand the processes behind speciation and biogeographical distributions. It is therefore perhaps not surprising that research devoted to systematics and phylogenetics on various branches of the tree of life has found increased interest over the last 10-20 years. Easier access to molecular data for use in phylogenetic analyses and the development of new analytical tools has promoted what has become a huge production of statistically testable hypotheses of evolutionary relationships.

Diptera is no exception and it was remarkable to see the number of papers dealing with phylogenetic analyses at the $\underline{7}^{\text {th }}$ International Congress of Dipterology in Costa Rica in August this year. In addition to being numerous, most of the phylogenetic studies that were presented at this conference used multiple markers, often in combination with morphological characters, to test for congruence and investigate the evolution of morphological and ecological traits. Datasets comprising more than 2000 characters were not uncommon and some even approached 6000! It is obvious in which direction phylogenetic research is going; what was regarded as a thorough study with a couple of genes yesterday will be a small pilot study tomorrow. - So also for the Chironomidae.

But, as for other dipteran taxa, morphological characters in phylogenetics are far from dead. As an example, Art Borkent at the Diptera Congress presented new evidence from pupal morphology that chironomids should be regarded as sister to all the other families in the Culicomorpha. The relationship has been backed by Pete Cranston et al.'s molecular phylogeny using molecular characters from four different genes. The latter study also has revealed Buchonomyiinae as sister to all other Chironomidae subfamilies, a relationship conforming to Murray and Ashe's earlier observation on the general plesiomorphous morphology of adult, pupa and egg of Buchonomyia thienemanni (Murray \& Ashe 1981; Ashe \& Murray 1983). There are many groups with unresolved relationships, however, and there will definitely be many interesting results from phylogenetic studies within our family in the years to come.

I hope that some of these will be presented at the next Chironomidae symposium in Trondheim and that they in concert with research results in taxonomy, ecology, genetics, cytology, palaeolimnology and toxicology will guarantee for a successful meeting. Please read more about the symposium and how to preregister in the Short Communications section in this newsletter. We look forward to see you there!

Torbjørn Ekrem, Museum of Natural History and Archaeology, Norwegian University of Science and Technology, Norway.E-mail: Torbjorn.Ekrem@vm.ntnu.no

\section{References}

Ashe P., Murray D. A. 1983. Observations on and descriptions of the egg-mass and eggs of Buchonomyia thienemanni Fitt. (Diptera: Chironomidae). In: Roback S. S., editors. Proceedings of the 8th International Symposium on Chironomidae. Philadelphia: Mem. Am. Entomol. Soc. 34, p 3-13.

Murray D., Ashe P. 1981. A description of the pupa of Buchonomyia thienemanni Fittkau, with notes on its ecology and on the phylogenetic position of the subfamily Buchonomyiinae (Diptera, Chironomidae). - Spixiana 4: 55-68. 